\title{
Evaluación de la variabilidad climática en el cantón Chillanes mediante los parámetros de la precipitación y la temperatura
}

DOI: https://doi.org/10.33262/ap.v3i4.1.125

(c) (i) (3) (2)

Evaluation of the climatic variability in the Chillanes canton through the parameters of precipitation and temperature

Franz Patricio Verdezoto-Mendoza. ${ }^{1}$, Juan Carlos Muyulema-Allaica. ${ }^{2}$, Anita Karina Serrano-Castro. ${ }^{3}, \&$ Luis Fernando Verdezoto-del-Salto. ${ }^{4}$

\begin{abstract}
Introduction: Climate variability in recent decades has intensified in the South American region, making it imperative to explore adequate adaptation and mitigation strategies to offset its current and future adverse impacts. Objective: To evaluate the climatic variability in the Chillanes canton located in the Hoya del Chimbo, Bolívar province, Ecuador, through the parameters of precipitation and temperature. Methodology: We used data extracted from monthly meteorological series of precipitation and temperature from the Chillanes-M130 Climate Station of the National Institute of Meteorology and Hydrology (CSNIMH), period 1989 - 2015. The series showed missing data that were completed using a univariate method of the difference criterion and later the climatic anomaly was verified by calculating the climatic anomalies of precipitation and temperature. Results: The analysis carried out in the 27 years did not show rainfall

\footnotetext{
${ }^{1}$ Universidad Estatal de Bolívar (UEB), Facultad de Ciencias Agropecuarias, Recursos Naturales y del Ambiente. Guaranda, Ecuador. email: fverdezoto@ueb.edu.ec ORCID ID: https://orcid.org/0000-00020049-005X

${ }^{2}$ Universidad Estatal Península de Santa Elena (UPSE), Facultad de Ciencias de la Ingeniería. La Libertad, Ecuador. email: jmuyulema@upse.edu.ec ORCID ID: https://orcid.org/0000-0002-9663-8935

${ }^{3}$ Universidad Estatal de Bolívar (UEB), Facultad de Ciencias de la Salud y del Ser Humano. Guaranda, Ecuador. email: aserrano@ueb.edu.ec ORCID ID: https://orcid.org/0000-0002-0347-1823

${ }^{4}$ Universidad Estatal de Bolívar (UEB), Facultad de Ciencias Agropecuarias, Recursos Naturales y del Ambiente. Guaranda, Ecuador. email: lverdezoto@ueb.edu.ec ORCID ID: https://orcid.org/0000-00028068-331X
} 
anomalies examined at $95 \%$ confidence with respect to the mean, therefore, it is considered a stable behavior despite the existence of natural phenomena of strong rainfall intensity such as the El Niño phenomenon in 1997. Conclusions: The temperature presented a positive anomaly that reveals that the annual average temperature is above the historical average recorded in the 27 years of study, showing an increase in temperature of $0.8^{\circ} \mathrm{C}$, which indicates that with respect to the temperature there is a climatic variability in the place of study.

Keywords: climate; variability; precipitation; temperature.

\section{Resumen}

Introducción: La variabilidad climática en las últimas décadas se ha intensificado en la región de Sudamérica, lo que hace imperativo explorar estrategias adecuadas de adaptación y mitigación para compensar sus impactos adversos actuales y futuros. Objetivo: Evaluar la variabilidad climática en el cantón Chillanes situado en la Hoya del Chimbo, provincia de Bolívar, Ecuador, mediante los parámetros de la precipitación y la temperatura. Metodología: Se utilizó datos extraídos de series mensuales meteorológicos de precipitación y temperatura de la Estación Climática Chillanes-M130 del Instituto Nacional de Meteorología e Hidrología (INAMH), periodo 1989 - 2015. La serie exteriorizó datos faltantes que fueron completados utilizando un método univariado del criterio de las diferencias y posteriormente se verificó la anomalía climática mediante el cálculo de las anomalías climáticas de la precipitación y la temperatura. Resultados: El análisis ejecutado en los 27 años no exteriorizó anomalías de la precipitación examinado a un $95 \%$ de confianza respecto a la media, por lo tanto, se considera un comportamiento estable a pesar de la existencia de fenómenos naturales de intensidad fuerte de precipitaciones como el fenómeno El Niño en el año de 1997. Conclusiones: La temperatura presentó una anomalía positiva que revela que la temperatura promedio anual está por encima del promedio histórico registrado en los 27 años de estudio, exteriorizando un incremento de la temperatura de $0,8^{\circ} \mathrm{C}$, lo que indica que respecto a la temperatura existe en el lugar de estudio una variabilidad climática.

Palabras clave: clima; variabilidad; precipitación; temperatura.

\section{Introducción}

Ecuador es un país altamente vulnerable a factores externos de diversa índole debida a su ubicación geográfica, que van desde eventos de origen natural o a actuaciones androgénicas (Oñate-Valdivieso et al., 2021; Villacís et al., 2020). Las consecuencias del cambio climático o la intensificación de fenómenos de variabilidad natural, como El Niño-Oscilación del Sur (ENOS), inciden de forma adversa en el desarrollo del país ( Serrano-Castro et al., 2019). Al igual que para la región, los escenarios proyectados de clima futuro realizadas en el marco de la Tercera Comunicación Nacional de Cambio 
Climático (TCN), muestran que de mantenerse la tendencia actual de la temperatura, el cambio que podría esperarse en Ecuador sería un aumento aproximado de $2{ }^{\circ} \mathrm{C}$ a finales del siglo XXI; e incluso, la Amazonía y Galápagos podrían presentar incrementos muy superiores Ministerio del Ambiente del Ecuador ([MAE], 2017). En términos específicos a escala país se observa que el cambio en la temperatura media para el periodo 2011-2040 estaría entre 0,6 y $0,75^{\circ} \mathrm{C}$, presentándose mayores incrementos en la costa $\left(0,7-0,9^{\circ} \mathrm{C}\right)$, Amazonía $\left(0,75-0,9^{\circ} \mathrm{C}\right)$ y Galápagos $\left(0,75-1{ }^{\circ} \mathrm{C}\right)$. Para mitad de siglo, el incremento estaría entre 0,9 y $1,7^{\circ} \mathrm{C}$, observándose los mayores cambios en la Amazonía (1,3 - 2,1 $\left.{ }^{\circ} \mathrm{C}\right)$ y Galápagos $\left(1,2-2,5^{\circ} \mathrm{C}\right)$. Finalmente, para el período 2071-2100, la temperatura media del país se incrementaría entre 0,9 y $2,8^{\circ} \mathrm{C}$, sin embargo, la Amazonía y Galápagos presentarían incrementos superiores, del orden de $1,3-3,5{ }^{\circ} \mathrm{C}$ y $1,2-4,4{ }^{\circ} \mathrm{C}$, respectivamente (MAE, 2017).

La variabilidad climática se refiere a variaciones en las condiciones climáticas medias, y otras estadísticas del clima en todas las escalas temporales y espaciales que se extienden más allá de la escala de un fenómeno meteorológico en particular (Blackmore et al., 2021; Mills-Novoa et al., 2020). A través del tiempo el clima presenta ciclos o fluctuaciones de diversa duración (Getahun et al., 2021). En diferentes años, los valores de las variables climatológicas como la temperatura y la precipitación fluctúan por encima o por debajo de lo normal, condición representada por el valor promedio de una variable climatológica en un período de por lo menos 30 años; la secuencia de estas oscilaciones alrededor de los valores normales, se conoce como variabilidad climática y su valoración se logra mediante la determinación de las anomalías que es la diferencia resultante entre el valor de la variable climatológica y su valor promedio en el tiempo (Instituto de Hidrología, Meteorología y Estudios Ambientales ([IDEAM -UNAL], 2018).

La Nacional Oceanic and Atmospheric Administration ([NOAA], 2021), realiza un listado desde 1950 en los cuales se ha detectado el Fenómeno El Niño a nivel del Pacífico y ha establecido criterios generales para clasificar la intensidad del fenómeno que puede ser: débil, moderado o fuerte, en cuanto a la amenaza, lo que depende del grado de calentamiento promedio (anomalía de cinco meses seguidos) en una zona identificada en el océano Pacífico Centro-Oriental llamada Zona Niño 3.4, lo que se determina por el índice ONI, el más reconocido actualmente y que se usa para su seguimiento y estudio. La variación del índice ONI, menciona que desde el año 1950 hasta el 2016 se han presentado 22 Fenómenos El Niño: 8 de intensidad débil; 8 de intensidad moderada y 6 de intensidad fuerte. Los eventos de los años 1997-1998 y 2014- 2016 han sido los más fuertes, si se tiene en cuenta el índice ONI (NOAA, 2021). Gil \& López (2011) exteriorizan que en los estudios de variabilidad temporal del clima es importante determinar sistemáticamente el sentido y evolución de las anomalías positivas y negativas, tanto térmicas como pluviométricas respecto a la media. Por lo tanto, un estudio evolutivo y estacional de las anomalías permite entender correctamente el comportamiento de estos climas y las posibles variaciones que puedan sufrir.

El presente estudio tiene como objetivo evaluar la variabilidad climática en el cantón Chillanes, ubicada en la hoya del Chimbo, provincia de Bolívar, Ecuador, para lo cual se 
ha analizado los datos meteorológicos de Presión y Temperatura atmosféricas entregados por la Estación Climática M130 (Chillanes del Instituto Nacional de Hidrología y Meteorología [INAMHI], 2016), en las coordenadas geográficas de Latitud -1,975556 y Longitud -79,063333, a una altitud de $2330 \mathrm{msnm}$.

\section{Figura 1}

\section{Ubicación de la Estación Meteorológica Chillanes M130 del INAMHI}

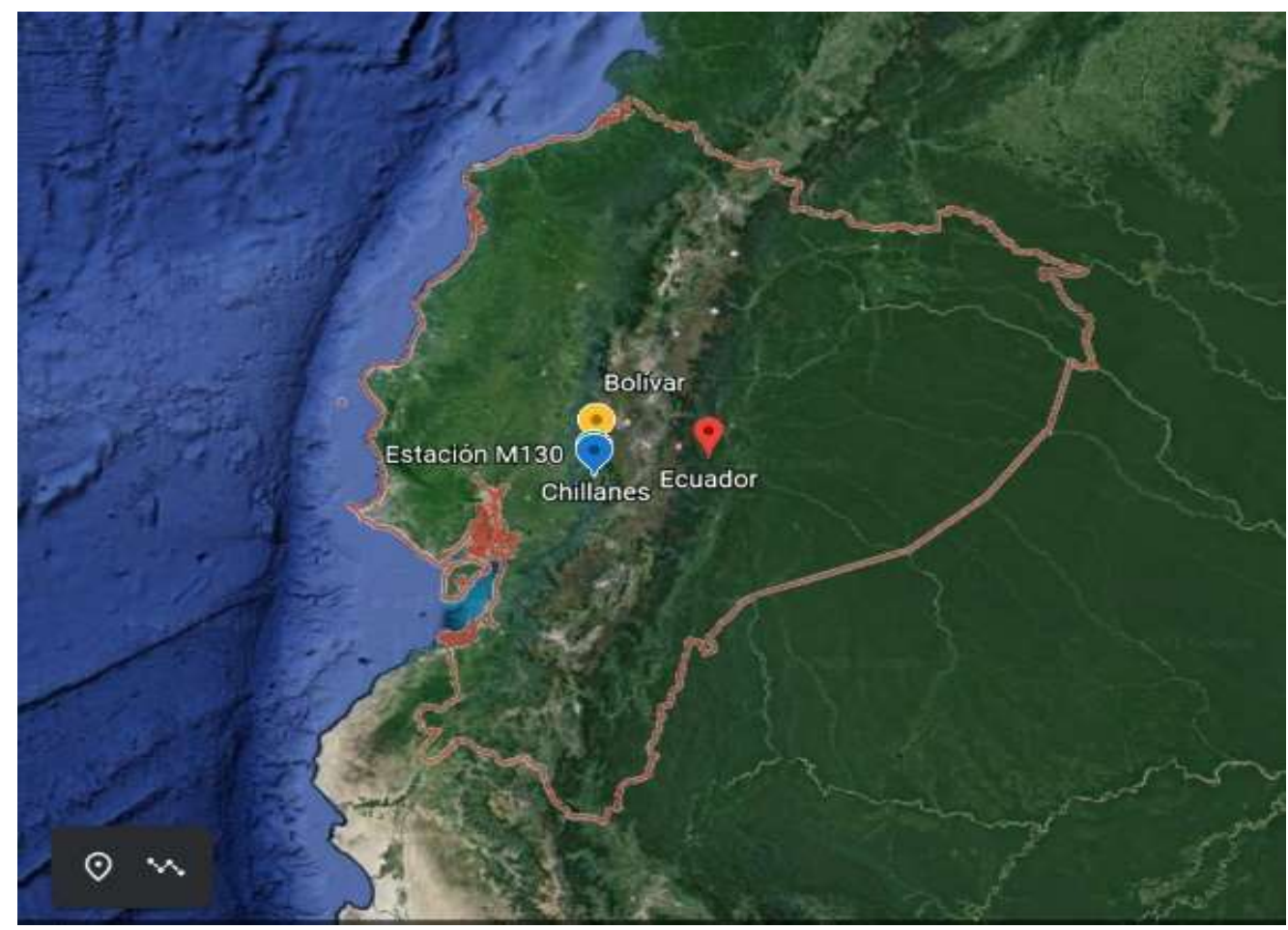

Nota: ubicación geográfica que muestra la variabilidad climática en el cantón

Chillanes, ubicada en la Hoya del Chimbo, provincia de Bolívar, Ecuador

Fuente: Los autores con base en Verdezoto (2017)

\section{Metodología}

La Organización Mundial de Meteorología ([OMM], 2017), estable un registro mínimo con extensión de 30 años para estadísticas climáticas confiables. Autores como Gil \& López (2011) han conseguido buenos resultados sobre posibles anomalías con 25 años de datos. En esta investigación se manejó series de datos mensuales de precipitación y temperatura para 27 años. Los datos aprovechables provienen de los registros de la estación del INAMHI Chillanes de la Provincia Bolívar, identificada con el código M0130, para el periodo 1989 - 2015.

La estación meteorológica Chillanes tiene datos faltantes que fueron rellenados a partir de la propia serie climática usando el método univariado del criterio de las diferencias (Barrera, 2004). Este método de relleno resultó ser adecuado, ya que se tenía información de todos los años de manera consecutiva y, los datos faltantes estaban repartidos esporádicamente a través de la serie. Los datos faltantes representaron menos del 10\% del total de datos esperados en las dos variables climáticas: temperatura, $9.3 \% \mathrm{y}$ 
precipitación, 4.3\%. El método univariado del Criterio de las Diferencias, consiste en sustituir la falta de un registro mensual por el valor del mes anterior, añadiendo el valor medio de las diferencias entre el mes anterior y el mes en cuestión (Gil \& López, 2011). En la ecuación 1 se muestra la relación usada para estimar los rellenos.

$$
C D=x \pm(x \pm x i) \quad \text { Ec. } 1
$$

Dónde:

CD: es el valor mensual obtenido por el Criterio de las diferencias.

$\mathbf{x}$ : es el valor mensual anterior al dato(s) faltante(s)

xi: es el valor medio mensual del mes antes de rellenar al dato(s) faltante(s).

Posterior al relleno, se procedió a trabajar con límites máximos y mínimos de confianza al 95\% del error estándar de la media y las barras de error estándar para cada conjunto de datos anual de la precipitación acumulada.

Para el cálculo de las anomalías, según Márdero et al. (2012) las anomalías en la precipitación y temperatura son la diferencia del parámetro observado en un periodo específico "i" (año, mes, día) de (Xi), respecto al promedio de las observaciones en un período determinado $(\mathrm{X})$, para la precipitación $\mathrm{P}$ la ecuación sería.

$$
\text { Anomalía }=\left(P_{i}-\bar{P}\right)(\text { en } \mathrm{mm}) \quad \boldsymbol{E c} .2
$$

Dónde: Pi precipitación para el período i

$\bar{P}$ Precipitación promedio histórica

Las anomalías también se expresan en porcentaje, lo cual indica al déficit o superávit de precipitación respecto al promedio:

$$
\text { \%Anomalía. }=\left(\frac{P i-\bar{P}}{\bar{P}}\right) * 100(\text { en \%) } \quad \text { Ec. } 3
$$

\section{Resultados}

\section{Precipitación}

La figura 2 muestra la precipitación acumulada anual desde el año 1989 hasta el año 2015, con una media de 837,4 mm y, una desviación estándar de 193,1 mm. En la figura se colocan los límites máximos y mínimos de confianza al 95\% del error estándar de la media y las barras de error estándar para cada conjunto de datos anual de la precipitación acumulada. Estas barras de errores muestran una longitud corta que indica una confiabilidad alta en la media calculada. Se destaca que los años 2008 y 1990 están muy cerca del límite de confianza adoptado.

A partir de la figura 2 podemos afirmar que en los 27 años de estudio no se observaron anomalías de la precipitación, porque las precipitaciones acumuladas anuales se encuentran dentro de los límites máximos y mínimos que representan el 95\% de confianza 
de error estándar de la media. Por lo expuesto, el acumulado anual de la precipitación muestra un comportamiento estable a pesar de la existencia de fenómenos naturales de intensidad fuerte de precipitaciones como el Niño del año 1997. El reporte de la NOAA (2021), indica que "los años 1993, 1997 y 2007 corresponden al fenómeno de El Niño, de los cuales el 1997 fue de una intensidad fuerte". Con lo cual, pareciera que en el callejón interandino no existiera una relación observable de las precipitaciones con el fenómeno de El Niño. Aceituno-Gutiérrez (1998), también indica algo similar al señalar que "en estas zonas no parece haber relación entre el fenómeno de El Niño y las precipitaciones anuales. Aún con episodios extraordinarios como el de 1982 - 83, las precipitaciones que se presentaron en esas áreas no tuvieron anomalías excepcionales". La estructura de la cordillera parece disipar la señal del fenómeno de El Niño actuando como una barrera natural, modulando la circulación atmosférica que inhibe la convección sobre las zonas de montaña (Samaniego et al., 2015).

\section{Figura 2}

Línea de tiempo de la precipitación acumulada anual periodo (1989 - 2015) con niveles de confianza al $95 \%$ de error estándar de la media

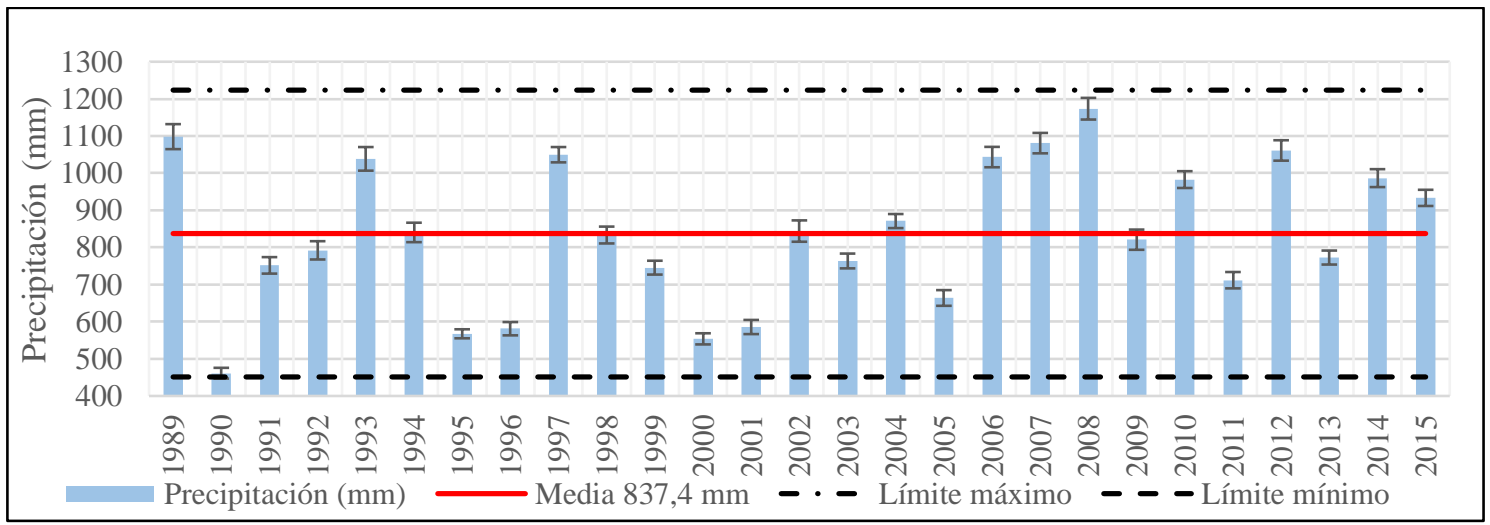

Nota: precipitaciones acumuladas anuales se encuentran dentro de los límites máximos y mínimos que representan el $95 \%$ de confianza de error estándar de la media.

Fuente: Los autores con base en Verdezoto (2017)

Aun cuando no pareciera existir una variabilidad climática en la precipitación y definir una estabilidad con un nivel de confianza del $95 \%$, es importante analizar que no todos los años son iguales y las precipitaciones presentan diferencias a considerar. En la

Figura 3 se presentan las anomalías de las precipitaciones para los 27 años propias de las condiciones particulares del clima en la zona andina. Al respecto Pourrut (1995), menciona “...en el callejón interandino, existe un régimen de tipo ecuatorial con dos estaciones lluviosas que corresponden a la penetración mayoritaria de masas respectivamente de aire amazónico en octubre y noviembre, o pacífico de enero a mayo (desplazamiento de la Zona de Convergencia Intertropical ZCIT. régimen de los vientos), siendo los totales pluviométricos variables según la altura, la exposición de las vertientes o la existencia de relieves transversales que definen cuencas más abrigadas y por lo tanto más secas", lo que quiere decir que por la topografía, las diferentes masas de aire que 
pueden ingresar a la hoya del Chimbo por los lados oriental y occidental de la cordillera andina, dan como resultado diferencias en los niveles de precipitación según cada año, proporcionándole características propias a la zona.

\section{Figura 3}

Porcentaje de anomalía de la precipitación en el periodo (1989 - 2015)

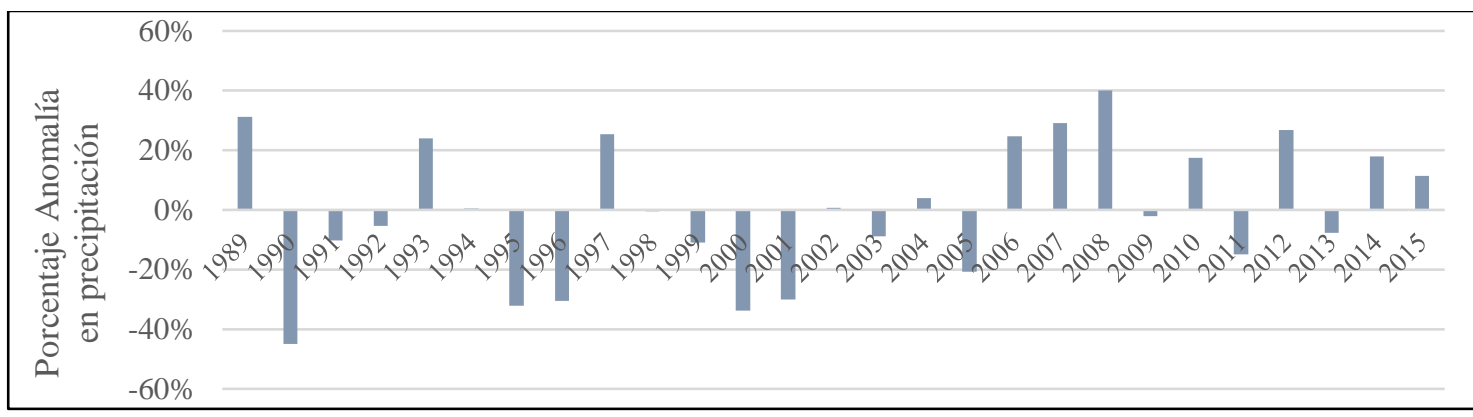

Nota: anomalías de las precipitaciones para los 27 años propias de las condiciones particulares del clima en la zona andina. Fuente: Los autores con base en Verdezoto (2017)

La figura 4 muestra la tendencia de la precipitación para el período de estudio, por medio del método de suavizado mediante media móvil a 5 años y ajustado a una ecuación lineal. En ella se observa una evolución ligeramente ascendente de la precipitación en el ajuste lineal. En la curva suavizada se observan dos ciclos de aproximadamente de 7 años, precedidos por un periodo (hasta 1999) con ligeras variaciones con respecto a la tendencia lineal. El primer ciclo muestra una disminución de las precipitaciones hasta el año 2003, a partir de ahí empieza un periodo con mayores precipitaciones hasta el año 2010, cuando parecieran comenzar nuevamente a disminuir la cantidad de lluvia.

\section{Figura 4}

Tendencia y medias móviles a 5 años de la precipitación en los 27 años

$$
\text { periodo }(1989-2015)
$$

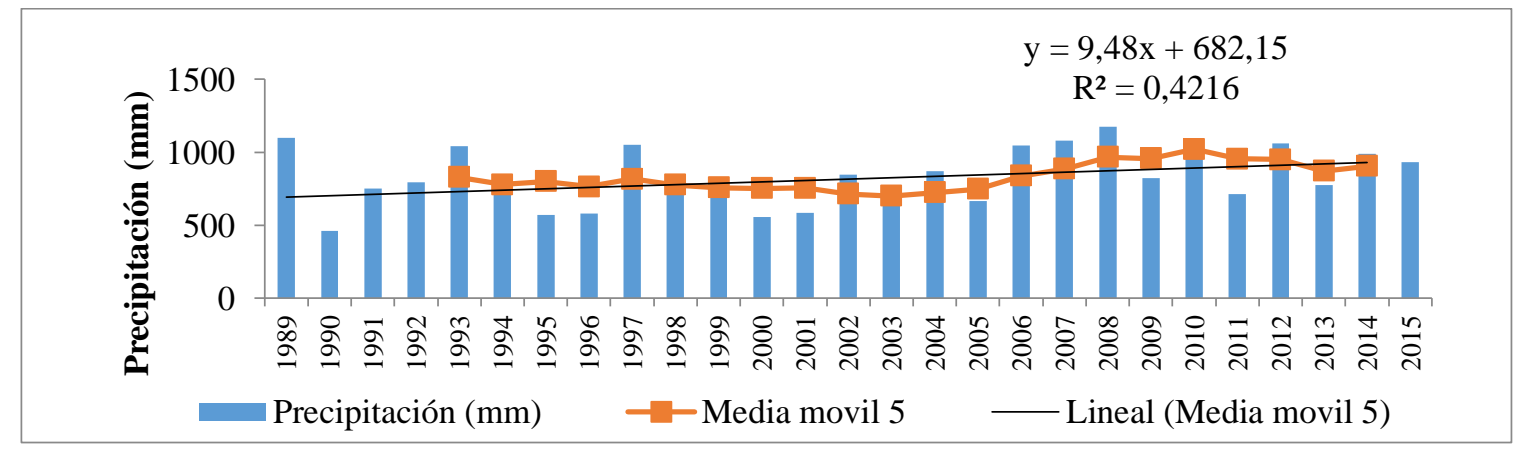

Nota: tendencia de la precipitación para el período de estudio, por medio del método de suavizado mediante media móvil a 5 años y ajustado a una ecuación lineal. Fuente: Los autores con base en Verdezoto (2017)

\section{Temperatura}

La figura 5 presenta el promedio de la temperatura anual desde el año 1989 hasta el año 2015 , con una media de $13,9^{\circ} \mathrm{C}$ y una desviación estándar de $0,4{ }^{\circ} \mathrm{C}$. Se colocan los 
límites máximos y mínimos de confianza al 95\% del error estándar de la media y las barras de error estándar de la precipitación acumulada en cada año. Estas barras de error presentan una longitud corta, lo que evidencia una confiabilidad alta en la media calculada. En la figura 5 se destaca que el año 2005 sobrepasa los niveles de confianza aceptada.

\section{Figura 5}

Línea de tiempo del promedio de temperatura anual periodo (1989 - 2015) con niveles de confianza al $95 \%$ de error estándar de la media

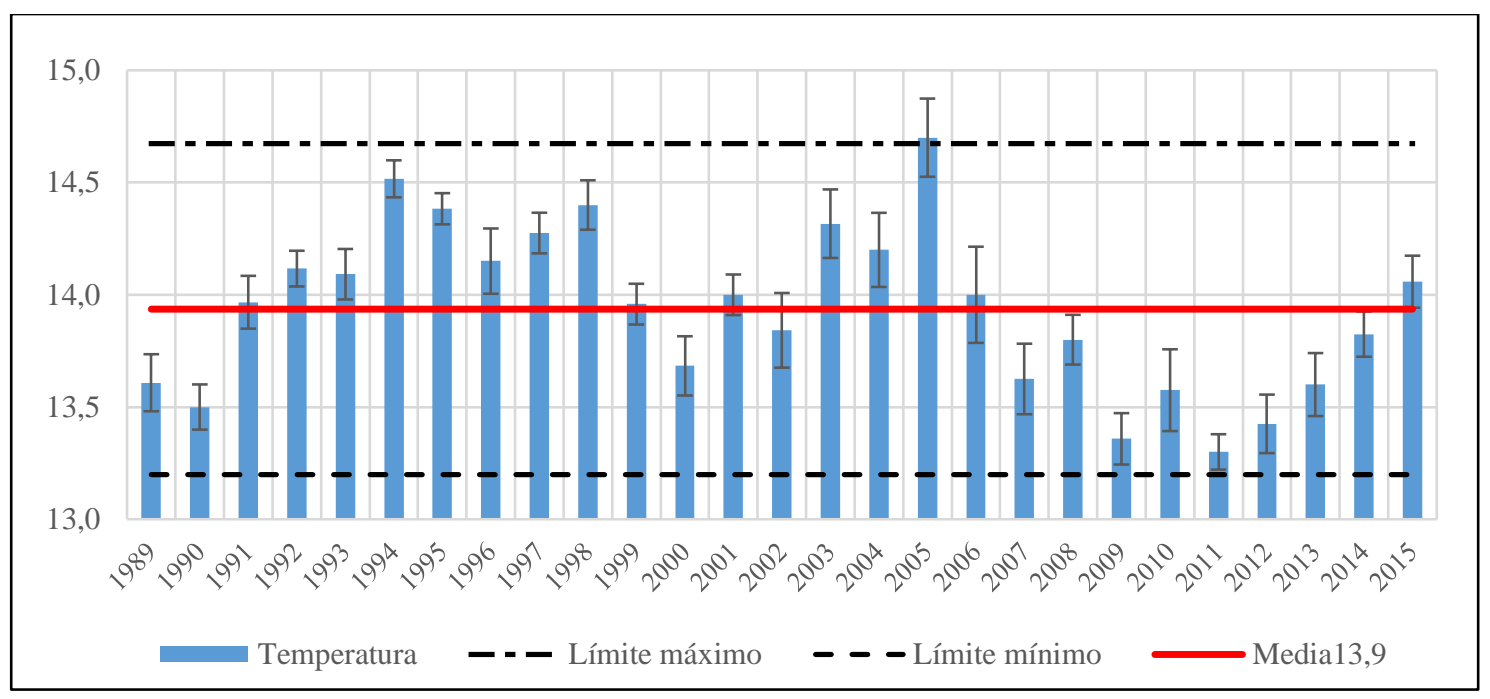

Nota: promedio de la temperatura anual desde el año 1989 hasta el año 2015 , con una media de $13,9^{\circ} \mathrm{C}$ y una desviación estándar de $0,4^{\circ} \mathrm{C}$.

Fuente: Los autores con base en Verdezoto (2017)

La figura 6 presenta las anomalías de la temperatura durante los 27 años de análisis. El año 2005 (marcado con rojo), presenta una anomalía positiva que indica que la temperatura promedio anual está por encima del promedio histórico registrado en los 27 años, con un incremento de la temperatura de $0,8{ }^{\circ} \mathrm{C}$ respecto a la media histórica. Considerando que solo existe un año anómalo de 27 , se constata una vez más que en el callejón interandino por su relieve característico, se genera un clima estable. La anomalía de la temperatura en el año 2005 puede estar sujeta a una serie de causas externas como: el Cambio Climático, alejadas de las condiciones de estabilidad propias de la zona Andina. El Instituto Nacional de Hidrología y Meteorología ([INAMHI], 2016) menciona al respecto que "La temperatura de la superficie terrestre y oceánica de la tierra está aumentando por encima de la media registrada durante el siglo XX. Un estudio de la Agencia Espacial Americana indica que el incremento de la temperatura afectó especialmente a la América Central, la mitad norte de Sud América. El Niño no es el único factor que está ocasionando altas temperaturas, puesto que este calentamiento continuo de la atmósfera terrestre tiene otro factor y es el acumulamiento de gases de efecto invernadero, lo cual favorece y favorecerá la ocurrencia de más años con récords térmicos en el futuro", o también puede ser producto de un error humano, falta de 
mantenimiento de la estación, pues no existe una causal natural extraordinaria el año 2005 en la zona de estudio.

\section{Figura 6}

Anomalía de la Temperatura en el periodo 1989 - 2015

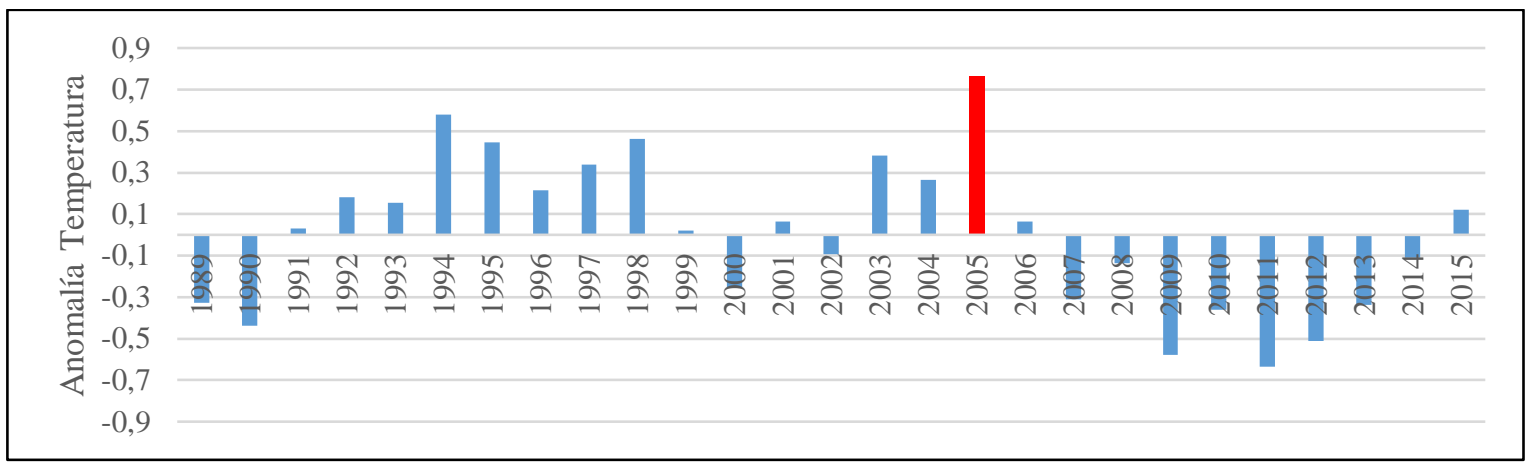

Nota: anomalías de la temperatura durante los 27 años de análisis.

Fuente: Los autores con base en Verdezoto (2017)

La figura 7 presenta una evolución ligeramente descendente de la temperatura suavizada mediante una media móvil a 5 años, la cual se ha ajustado por medio de una ecuación lineal. La curva de suavizado marca cuatro periodos de aproximadamente 7 años, con ligeras variaciones para mostrar cuatro ciclos: dos con aumento de temperaturas y dos ciclos con disminución, intercalados unos con otros. De esta manera, se constata una cierta estabilidad en el comportamiento de la temperatura de esa zona. Lo expuesto es confirmado por Pourrut (1995); Engel et al. (2014); Carrillo-Rojas et al. (2020); OñateValdivieso et al. (2021) cuando indican que la barrera natural de los Andes marca en los valles una cierta estabilidad del clima.

\section{Figura 7}

Tendencia y medias móviles a 5 años de la Temperatura en los 27 años periodo (1989-2015)

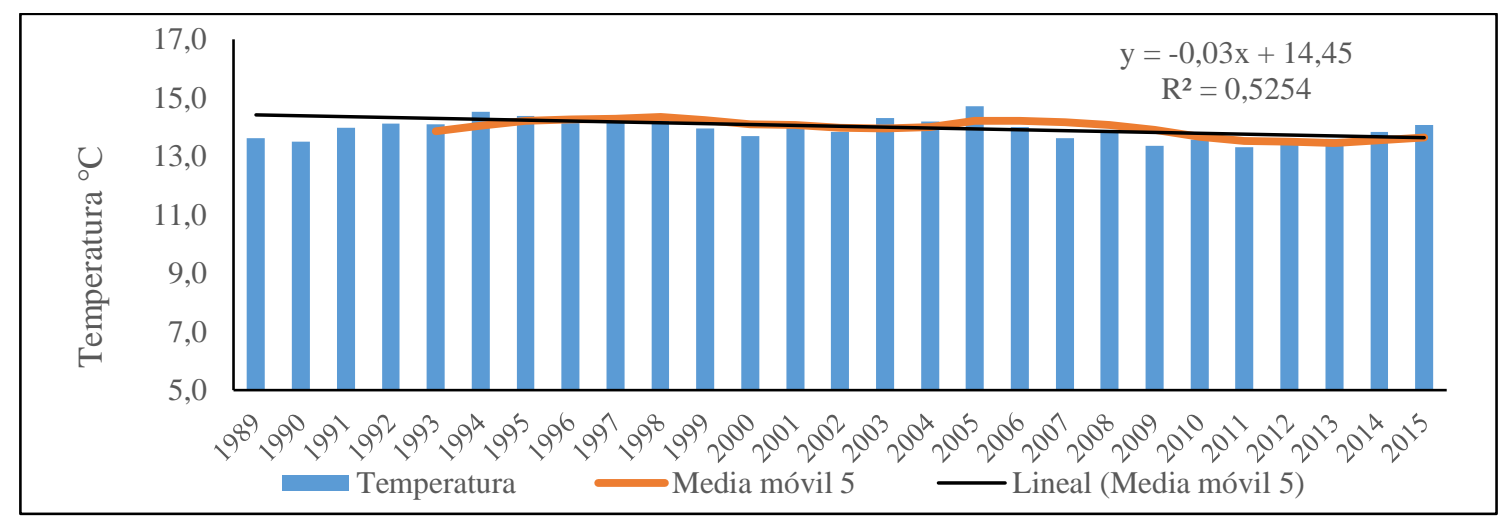

Nota: evolución ligeramente descendente de la temperatura suavizada mediante una media móvil a 5 años.

Fuente: Los autores con base en Verdezoto (2017)

La figura 8 presenta una comparativa de las medias móviles a 5 años de la precipitación y la temperatura. En ella se observa una correspondencia indirecta moderada entre periodos, con un coeficiente de correlación de Pearson de -0,6. En la figura se observa 
una disminución de precipitaciones y aumento de temperatura en los periodos 1993-1997 y 2010 - 2015. En el periodo 1998 - 2009 al disminuir las precipitaciones, disminuyen las temperaturas; y entre los años 2005 - 2009 aumentan las temperaturas y precipitaciones en simultáneo.

\section{Figura 8}

Comparativa de las medias móviles a 5 años de la precipitación y temperatura en el periodo 1989 - 2015

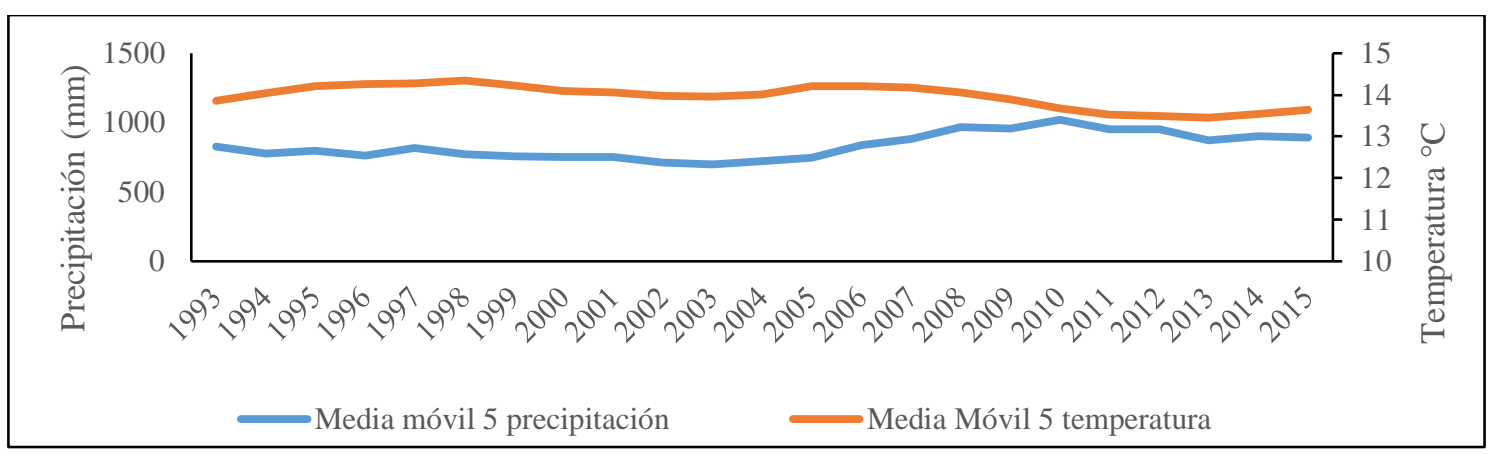

Nota: comparativa de las medias móviles a 5 años de la precipitación y la temperatura.

Fuente: Los autores con base en Verdezoto (2017)

Esta variedad de comportamientos se debería a las características geomorfológicas de la cordillera andina y la aceleración del cambio climático que al ser un fenómeno de escala global afecta al Ecuador, así como lo demuestra la Tercera Comunicación Nacional (MAE, 2017), con escenarios de aumentos de temperatura en todo el territorio nacional. En el área de estudio, al igual que el resto del mundo vive transformaciones en sus patrones de clima haciéndole vulnerable al cambio y variabilidad climática. Tirado (2010) menciona que los impactos del cambio climático serán ocurrencias de eventos extremos que traerán impactos negativos sobre la humanidad, como la precipitación que a su vez favorecen los deslizamientos e inundaciones, y la temperatura que lleva consigo riesgos de mortalidad, disminución del recurso hídrico, pérdida de cosechas, entre otros.

\section{Discusión}

De los datos analizados se evidencia un contraste entre la precipitación y la temperatura, por un lado la precipitación no presenta anomalías climáticas lo que conlleva a una percepción de estabilidad, a pesar de existir la presencia de fenómenos naturales como el ENOS, si bien esto es explicado por Pourrut (1995) debido a que geográficamente el área de estudio está dentro del callejón interandino, no se puede negar que existe evidencia de Cambio Climático reportado en el informe especial del Intergovernmental Panel on Climate Change ([IPCC], 2019) sobre los impactos del calentamiento global de $1,5^{\circ} \mathrm{C}$. En este estudio se ha probado que existe una anomalía climática de la temperatura en el año 2005 lo cual indica una variabilidad climática en el sector, además se evidencia que en el periodo de años del 2007 al 2009 aumentan las precipitaciones y temperaturas en simultaneo algo que no ocurre en años anteriores en donde las dos variables no son directamente proporcionales lo que hace pensar, que tal vez estos cambios están asociados a la aceleración del Cambio Climático y su efecto sobre el clima, pudiendo desencadenar una serie de riesgos sobre la población urbana y rural. Blackmore et al. 
(2021) realizó un estudio en la zona andina del Ecuador y menciona que uno de los principales factores que influyen en la vulnerabilidad de las personas es el aumento de la imprevisibilidad de las precipitaciones y el aumento de las temperaturas asociadas a la variabilidad climática provocando cambios en las temporadas de siembra y ha afectado negativamente los rendimientos de los cultivos.

Nuestros resultados evidencian una variabilidad climática en la zona de predominancia rural, por lo que se propone buscar estrategias para enfrentarla. Ponce (2020), menciona que la agricultura en las zonas rurales especialmente en las zonas frías andinas debe adaptarse a la variabilidad climática por medio de la diversificación de cultivos más tolerantes. Es evidente que estamos frente a un cambio climático y la variabilidad según las proyecciones del IPCC pueden incrementarse, por lo que es imprescindible el buen manejo de datos para generar información relevante que ayuden a tomar decisiones para disminuir un posible impacto negativo en la zona estudiada. Es recomendable plantearse diversos estudios sobre variabilidad climática en la zona, especialmente los que relaciones las diversas afectaciones en la agricultura, puesto que esta se verá afectada por el cambio climático debido al aumento de las temperaturas, los cambios en los patrones de precipitación y el aumento de las concentraciones de $\mathrm{CO}_{2}$ atmosférico. Los productores del sector pueden adaptarse al cambio ambiental cambiando la rotación, variando los fertilizantes, la labranza y otros insumos agrícolas. Sin embargo, hay pocas publicaciones que identifiquen combinaciones de insumos y diversidad de cultivos que mitiguen los efectos del cambio climático proyectado.

\section{Conclusiones}

- Del análisis de las anomalías climáticas de la precipitación acumulada mensual y la temperatura media mensual de Estación Climática Chillanes en el período de estudio (2010-2015), analizadas frente a los promedios históricos mensuales de 27 años (1999 - 2015) con un nivel de confianza del 95\%, se concluye que no existe variabilidad climática en el área de estudio a excepción de un hecho aislado en el 2005 correspondiente a una anomalía de la temperatura.

- La no variabilidad climática puede ser producto de la cordillera de los Andes que actúa como barrera topográfica sobre la región que se encuentra ubicada en la hoya lateral occidental del río Chimbo a $2600 \mathrm{msnm}$. Localmente esta barrera natural divide y aísla las masas de aire que provienen de los dos lados de la cordillera, separando la humedad que se origina en la cuenca Amazónica y en el Atlántico Tropical de las zonas secas áridas y semiáridas de la Costa o Litoral de Ecuador. Posiblemente, por esta razón, los fenómenos climáticos naturales como el de El Niño que, generan un gran impacto en la zona costera ecuatoriana, en esta zona Andina son casi imperceptibles, inclusive bajo eventos extraordinarios como el ocurrido en el año 1997.

- La anomalía de la temperatura del año 2015 no representa a alguna eventualidad natural extrema como El Niño, el mismo que en este año es calificado como "neutro", por lo que otros factores externos podrían haber incidido para la 
ocurrencia de esta anomalía, que puede ser de carácter natural o antrópica y de esta última puede haber incidido la forma de tomar y controlar los datos.

- Para los 27 años de estudio (1997-2015), la precipitación acumulada mensual presenta una ligera tendencia positiva que indica que las lluvias han aumentado levemente, pero esta tendencia no es un factor que genere una variabilidad climática marcada, posiblemente, debido a la barrera natural de los Andes. La tendencia de la temperatura promedio mensual muestra una disposición levemente negativa, indicando que existe cierta estabilidad y, al igual que la precipitación, está relacionada con la barrera natural de los Andes por lo que no genera una variabilidad climática fuerte que sobrepase los límites al 95\% de confianza con los que se trabajó.

- Las estaciones meteorológicas presentan vacíos en la data, los cuales pueden ser rellenados con diferentes métodos, en este caso hemos procedido a rellenar con métodos univariados porque los porcentajes de falta de datos eran menores al $10 \%$ de los datos leídos. Se recomienda que se utilicen diferentes métodos de relleno como el multivariado a fin de disminuir el error en los resultados.

\section{Referencias bibliográficas}

Aceituno-Gutiérrez, P. (1998). El Fenómeno El Niño 1997-98. Bulletin de l'Institut Français d'études Andines, 27(3).

Barrera, A. (2004). Técnicas de completado de series mensuales y aplicación al estudio de la influencia de la NAO en la distribución de la precipitación en España. GAMA, $15-19$.

Blackmore, I., Rivera, C., Waters, W. F., Iannotti, L., \& Lesorogol, C. (2021). The impact of seasonality and climate variability on livelihood security in the Ecuadorian Andes. In Climate Risk Management (Vol. 32). https://doi.org/10.1016/j.crm.2021.100279

Carrillo-Rojas, G., Schulz, H. M., Orellana-Alvear, J., Ochoa-Sánchez, A., Trachte, K., Célleri, R., \& Bendix, J. (2020). Atmosphere-surface fluxes modeling for the high Andes: The case of paramo catchments of Ecuador. Science of the Total Environment, 704, 135372. https://doi.org/10.1016/j.scitotenv.2019.135372

Engel, Z., Skrzypek, G., Chuman, T., Šefrna, L., \& Mihaljevič, M. (2014). Climate in the Western Cordillera of the Central Andes over the last 4300 years. Quaternary Science Reviews, 99(1), 60-77. https://doi.org/10.1016/j.quascirev.2014.06.019

Getahun, A. B., Ayal, D. Y., Ture, K., \& Zeleke, T. T. (2021). Determinants of climate variability adaptation strategies: A case of Itang Special District, Gambella Region, Ethiopia. Climate $\quad$ Services, 100245. https://doi.org/10.1016/j.cliser.2021.100245

Gil, S., \& López, F. (2011). Tendencia de las precipitaciones y temperaturas en una 
pequeña cuenca fluvial del sureste peninsular semiárido. Boletín de La Asociación de Geógrafos Españoles, 56, 349-371.

Instituto de Hidrología, Meteorología y Estudios Ambientales (IDEAM -UNAL). (2018). Variabilidad Climática y el cambio climático en Colombia. In Bogotá, D.C.

Instituto Nacional de Hidrología y Meteorología (INAMHI). (2016). Boletín Climatológico Anual 2015.

Intergovernmental Panel on Climate Change (IPCC). (2019). Calentamiento Global de $1,5^{\circ} \mathrm{C}$. In Intergovernmental Panel on Climate Change.

Márdero, S., Nickl, E., Schmook, B., Schneider, L., Rogan, J., Christman, Z., \& Lawrence, D. (2012). Sequías en el sur de la península de Yucatán: análisis de la variabilidad anual y estacional de la precipitación. Investigaciones Geográficas, 78, 19. https://doi.org/10.14350/rig.32466

Mills-Novoa, M., Boelens, R., Hoogesteger, J., \& Vos, J. (2020). Governmentalities, hydro social territories \& recognition politics: The making of objects and subjects for climate change adaptation in Ecuador. Geoforum, 115(July), 90-101. https://doi.org/10.1016/j.geoforum.2020.06.024

Ministerio del Ambiente del Ecuador (MAE). (2017). Tercera Comunicación Nacional del Ecuador.

National Oceanic and Atmospheric Administration (NOAA). (2021). Centro de Predicción Climática - ONI.

Organización Meteorológica Mundial (OMM). (2017). Directrices de la Organización Meteorológica Mundial sobre el cálculo de las normales climáticas. Organización Meteorológica Mundial, 21.

Oñate-Valdivieso, F., Oñate-Paladines, A., Armijos, J., \& Puchaicela, D. (2021). Regional relationships for bank full hydraulic geometry and discharge in the southern Andes of Ecuador: An application in the estimation of geomorphological $\begin{array}{llll}\text { runoff thresholds. } & \text { Geomorphology, } & 392, & 107914 .\end{array}$ https://doi.org/10.1016/j.geomorph.2021.107914

Ponce, C. (2020). Intra-seasonal climate variability and crop diversification strategies in the Peruvian Andes: A word of caution on the sustainability of adaptation to climate change. World Development, $127, \quad 104740$. https://doi.org/10.1016/j.worlddev.2019.104740

Pourrut, P. (1995). El Agua en el Ecuador (Corporación Editora Nacional (ed.)).

Samaniego, N., Eguiguren, P., Maita, J., \& Aguirre, N. (2015). Clima de la Región Sur el Ecuador: historia y tendencias. Biodiversidad Del Páramo: Pasado, Presente y Futuro Clima Clima, December, 43. 
Serrano-Castro, A. K., Muyulema-Allaica, J. C., Usca-Veloz, R. B., \& González-Rivera, M. M. (2019). Aplicación de la metodología STAR para el análisis del riesgo sísmico de la provincia Bolívar - Ecuador. Ciencia Digital, 3(4), 227-249. https://doi.org/10.33262/cienciadigital.v3i4.968

Tirado, M. (2010). Cambio climático y salud. Informe SESPAS 2010. Gaceta Sanitaria, 24(Suppl 1), 78-84. https://doi.org/10.1016/j.gaceta.2010.10.004

Verdezoto, M. F. (2017). Diagnóstico de la resiliencia al cambio y variabilidad climática en la ciudad de Guaranda. Guayaquil. Escuela Superior Politécnica del Litoral (ESPOL).

Villacís, A. G., Bustillos, J. J., Depickère, S., Sánchez, D., Yumiseva, C. A., TroyaZuleta, A., Barnabé, C., Grijalva, M. J., \& Brenière, S. F. (2020). Would tropical climatic variations impact the genetic variability of triatomines: Rhodnius ecuadoriensis, principal vector of Chagas disease in Ecuador? Acta Tropica, 209(April), 105530. https://doi.org/10.1016/j.actatropica.2020.105530

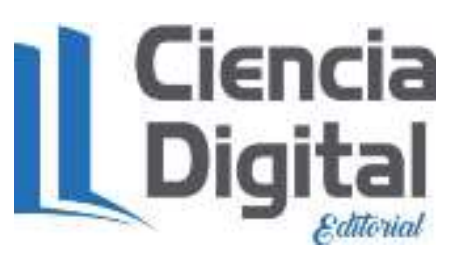




\section{PARA CITAR EL ARTÍCULO INDEXADO}

Verdezoto-Mendoza, F. P., Muyulema-Allaica, J. C., Serrano-Castro, A. K., \& Verdezoto-del-Salto, L. F. (2021). Evaluación de la variabilidad climática en el cantón Chillanes mediante los parámetros de la precipitación y la temperatura . AlfaPublicaciones, 3(4.1), 70-84. https://doi.org/10.33262/ap.v3i4.1.125

\section{ULiencia}

El artículo que se publica es de exclusiva responsabilidad de los autores y no necesariamente reflejan el pensamiento de la Revista Alfa Publicaciones.

El artículo queda en propiedad de la revista y, por tanto, su publicación parcial y/o total en otro medio tiene que ser autorizado por el director de la Revista Alfa Publicaciones.
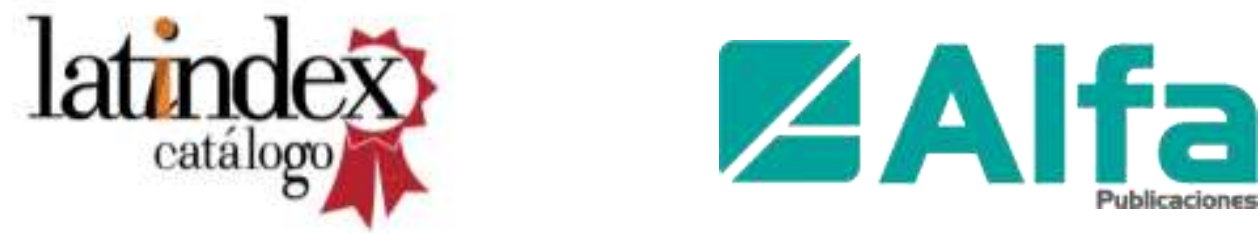